Check for updates

Cite this: J. Mater. Chem. B, 2017, 5, 9502

Received 24th October 2017,

Accepted 24th November 2017

DOI: $10.1039 / \mathrm{c} 7 \mathrm{tb} 02792 \mathrm{c}$

rsc.li/materials-b

\section{Guiding growth orientation of two-dimensional Au nanocrystals with marine chitin nanofibrils for ultrasensitive and ultrafast sensing hybrids $\dagger$}

\author{
Yijun Chen, ${ }^{\text {ab }}$ Zhengqin Liu, ${ }^{b}$ Mingjie Li, ${ }^{a}$ Xiaochen $\mathrm{Wu}^{a}{ }^{a}$ Jun You (D) ${ }^{a}$ and \\ Chaoxu Li (D)*a
}

\begin{abstract}
We demonstrate that marine chitin nanofibrils are able to modulate the growth direction of two-dimensional Au nanocrystals from nanoribbons, nanokites to nanosheets. The mechanism investigation reveals that deacetylation and exfoliation of chitin nanofibrils are essential to template directional growth of Au nanocrystals. The tight adhesion of chitin nanofibrils on the Au surface enables the design of functional hybrids as ultrafast and ultrasensitive responsive devices for humidity and pressure. Interestingly, the humidity responsive device shows an abrupt increase in resistance of up to 3 orders of magnitude with a tiny variation of relative humidity from $62 \% \mathrm{RH}$ to $63 \% \mathrm{RH}$, and was capable of precisely sensing speech. As a conductive filler, only 0.09 vol\% gold nanoribbons are able to append to tissue paper with a sheet resistance of up to $220 \Omega \mathrm{sq}^{-1}$, which can be used as a frequently-used pressure-sensing device. These sensing hybrids are highly promising in smart clothing and electronic skins.
\end{abstract}

Bio-composites in living organisms rely on biologic macromolecular assemblies to regulate the growth of inorganic crystals along specific crystallographic orientations and for diverse ondemand biophysical functions. ${ }^{1}$ The organic phases have two essential characteristics to achieve particular crystallo-chemical specificity of bio-mineralization, i.e. molecular complementarity of local binding sites (ionic functional groups) to molecularly recognize and bind inorganic ions on the organic interfaces, and molecular pre-organization (assembling nanostructures) for the spatial guidance of inorganic nucleation and growth orientation. A typical example is collagenous proteins in bone mineralization: ${ }^{2}$ collagen fibrils with positively charged regions provide infiltration sites for amorphous calcium phosphate, whose periodic charged domains further form nucleation sites for controlling apatite into parallel arrays of oriented crystal platelets. Analogously

\footnotetext{
${ }^{a}$ CAS Key Lab of Biobased Materials, Qingdao Institute of Bioenergy and Bioprocess Technology, Chinese Academy of Sciences, Qingdao 266101, China. E-mail: licx@qibebt.ac.cn,youjun@qibebt.ac.cn

${ }^{b}$ College of Textiles \& Clothing, Qingdao University, Qingdao 266071, P. R. China

$\dagger$ Electronic supplementary information (ESI) available. See DOI: 10.1039/c7tb02792c
}

to proteins, chitin exists as the second most abundant polysaccharide in nature and is a major organic component of several marine bio-composites such as nacre, the shells of cuttlefish Sepia officinalis, and the shells of Lingula unguis. These bio-composites in principle share a structural hierarchy in which chitin nanofibrils align with particular axes of laminated two-dimensional (2D) aragonite or apatite. ${ }^{3}$ However, with a relatively unitary chemical structure (i.e. linear polysaccharide chains of $\beta$-(1-4)2-acetamido-2-deoxy-D-glucopyranose residues) and a fibrous pre-organization structure, how chitin recognizes and mineralizes specific inorganic ions in 2D remains elusive, thus hindering its application in the synthesis of non-biologic nanomaterials and biomimetic hybrids.

Meanwhile, due to their fantastic size/morphology-dependent properties in electronics, optics, catalysis, sensing and biomedicine, $\mathrm{Au}$ nanocrystals have attracted rapidly increasing interest in nanotechnology. ${ }^{4,5}$ In contrast to $\mathrm{Au}$ nanoparticles, 2D Au nanocrystals with large aspect ratios (e.g. platelets, prisms, and belts) show their superiority in mechanical compliancy and low electrical percolation thresholds in biomimetic conductive hybrids, ${ }^{6,7}$ thus showing promise in applications such as flexible pressure sensors, " "on-off" switches and strain/force/vibration sensors. ${ }^{7}$ Their morphologies are conventionally controlled in "kinetically controlled" reducing reactions of wet chemistry in the presence of soft templates and capping agents. ${ }^{5-9-11}$ For example, Au nanoplates and nanobelts were synthesized using a template of lyotropic liquid crystal phases. ${ }^{12}$ Au nanobelts and nanocombs were synthesized in the presence of ascorbic acid and mixed surfactants, ${ }^{13}$ while nanokites and microplates were synthesized solely in the presence of poly(vinyl pyrrolidone). ${ }^{14}$ In order to achieve an environment-friendly synthesis, the bio-mineralization of biomolecules (e.g. $\operatorname{proteins}^{15}$ and DNA) and their assemblies (e.g. amyloid fibrils ${ }^{6}$ ) has been used to synthesize $\mathrm{Au}$ nanosheets. ${ }^{16}$ The abundant ionic groups of proteins and their tertiary structures were believed to ensure the binding of $\mathrm{AuCl}_{4}{ }^{-}$or $\mathrm{Au}(\mathrm{CN})_{2}{ }^{-}$and control the growth of $\mathrm{Au}$ nanocrystals. ${ }^{17}$ In spite of these advances, it still remains challenging to tune the morphologies of $\mathrm{Au}$ nanocrystals 
through the bio-mineralization of individual biological molecules for various on-demand applications.

Herein we show that chitin nanofibrils were able to not only bind and reduce chloroauric ions on their surfaces, but also to guide the growth direction of 2D Au nanocrystals into various geometries (i.e. nanosheets, nanokites and nanoribbons). This bio-mineralization relies strongly on the deacetylation degree and exfoliation level of the chitin nanofibrils, where the former offers essential binding and reducing capacities of $\mathrm{AuCl}_{4}{ }^{-}$ions in a "kinetically controlled" pathway, while the latter offers cooperative topographical templates for the nucleation and oriented crystallization of Au nanocrystals. In particular, the synthesized 2D Au nanocrystals had their $\{111\}$ crystallographic facets capped with a tuneable amount of chitin nanofibrils. When organizing Au nanosheets into hybrid films with layer-bylayer structures, the intercalated layer of chitin nanofibrils may endow the hybrids with metallic conductivity unprecedentedly sensitive to environmental humidity. When filtering in Au nanoribbons into cellulose paper, the chitin nanofibrils had strong interactions with the polyhydroxy surfaces and thus enabled strong adhesion of the Au nanoribbons to the cellulose fibres. The resultant hybrid not only had an ultra-low percolation threshold of $\mathrm{Au}$ for metallic conductivity, but also had a sensitive and rapid conductivity response to applied pressure.

Chitin exists naturally in crustacean exoskeletons and mollusc shells in the form of uncharged and aligned nanofibrils (Fig. 1A). However it could be positively charged through partial deacetylation under base conditions. ${ }^{18}$ For instance, $\sim 10 \%$ degree of deacetylation was achieved by incubating chitin in 33 wt $\%$



Fig. 1 Pathway followed to guide the growth direction of Au nanocrystals with chitin nanofibrils. (A) Chitin nanofibrils in crab shells and their chemical structure. (B) Chitin deacetylation. (C) CNF-bundles produced using mechanical exfoliation. (D) Au nanoribbons produced from CNF-bundles (0.01 wt\%) and chloroaurate $(0.4 \mathrm{mM})$ at $\mathrm{pH} 3.5$. (E) Au nanokites produced from CNFbundles (0.04 wt\%) and chloroaurate $(0.4 \mathrm{mM})$ at $\mathrm{pH}$ 3.5. (F) A typical AFM image of an Au nanokite. (G) A typical AFM image of an Au nanoribbon. (H) CNFs produced using mechanical and ultrasonic exfoliation. (I and J) $\mathrm{Au}$ nanosheets produced from CNFs (0.08 wt\%) and chloroaurate ions $(0.4 \mathrm{mM})$ at $\mathrm{pH}$ 2. (K) Height profiles of Au nanocrystals along the indicated lines in ( $F, G$ and $J)$.
$\mathrm{NaOH}$ at $90{ }^{\circ} \mathrm{C}$ for $2 \mathrm{~h}$ (Fig. 1B). The obtained amino groups on the chitin nanofibrils have a $\mathrm{p} K_{\mathrm{a}}$ value of 6.3 , and under acidic conditions not only offered sufficient binding sites for negatively charged ions, but were also advantageous in exfoliating the chitin nanofibrils through either mechanical agitation (1300 rpm for 7 days) or a combination of mechanical agitation (1300 rpm for 2 days) and ultra-sonication ( $400 \mathrm{~W}$ for $0.5 \mathrm{~h}$ ). The chitin nanofibrils were partially exfoliated in the form of fibrous bundles (CNF-bundles, Fig. 1C and Fig. S1A, ESI $\dagger$ ) when following the former exfoliation procedure, while being relatively completely exfoliated in the form of individual fibrils when following the latter exfoliation procedure (CNFs, Fig. 1H \& Fig. S1B, ESI $\dagger$ ). Having the same deacetylation degrees and zetapotential (Fig. S2, ESI $\dagger$ ), both types of chitin nanofibril maintained stability in acidic solutions and did not show clear sedimentation within a period of hours, in sharp contrast to the rapid sedimentation of pristine chitin (Fig. S3, ESI $\dagger$ ). The CNFs were individually dispersed in the solution with an average diameter of $\sim 14.0 \mathrm{~nm}$ and an average length of $\sim 336 \mathrm{~nm}$ (Fig. S4A-C, ESI $\dagger$ ). The CNF-bundles consisted of partially exfoliated chitin nanofibrils, being highly branched and entangled with a diameter of $25-150 \mathrm{~nm}$ and a length of $0.5-5.5 \mu \mathrm{m}$ (Fig. S4D and E, ESI $\dagger$ ).

When adding chloroauric acid under acidic conditions, chloroauric ions may be mineralized into $\mathrm{Au}$ nanomaterials by CNF-bundles and CNFs at higher temperatures (e.g. $\left.90{ }^{\circ} \mathrm{C}\right)$. Even in the presence of the same $\mathrm{AuCl}_{4}{ }^{-}$concentration (e.g. $0.4 \mathrm{mM}$ ), different morphologies of $\mathrm{Au}$ nanomaterials were obtained by varying the exfoliation states and concentrations of the chitin nanofibrils (Fig. S5A-C, ESI $\dagger$ ). In the case of CNFbundles at lower concentrations (e.g. $0.01 \mathrm{wt} \%$ ), Au nanoribbons were synthesized with widths up to $200 \mathrm{~nm}$ and aspect ratios up to 50 (Fig. 1D, G and K). At higher concentrations of CNF-bundles (e.g. $0.04 \mathrm{wt} \%$ ), Au nanokites were synthesized and featured a nanosheet head with a micrometric lateral length and a nanoribbon tail with a length over $10 \mu \mathrm{m}$ (Fig. 1E, F and K). In the case of CNFs, Au nanosheets were synthesized with a lateral size tuneable to as high as $10^{1} \mu \mathrm{m}$ by the concentration of CNFs (Fig. 1I-K and Fig. S5D-F, ESI $\dagger$ ). Their height AFM profiles further indicate an increasing thickness from Au nanoribbons to nanokites and nanosheets (Fig. 1K), and their XRD spectra confirm the 2D growth mechanism with predominantly exposed $\{111\}$ crystallographic surfaces (Fig. S6, ESI $\dagger)^{19}$

In contrast to inert pristine chitin nanofibrils (Fig. S7, ESI $\dagger$ ), the positively charged chitin nanofibrils during the bio-mineralization processes not only bound and reduced chloroauric ions on their surfaces as the reducing agent, but also guided the growth orientation of $2 \mathrm{D}$ Au nanocrystals as the capping agent. In the homogenous solution of CNFs, there exist relatively homogeneous distributions for the reducing and capping effects. $\mathrm{Au}$ nanosheets were synthesized analogously to those in a solution of amyloid fibrils, ${ }^{6,7}$ through a "kinetically controlled" nucleation-and-growth mechanism. ${ }^{20}$ As shown in Fig. 2A and Fig. S8A, B (ESI $\dagger$ ), chloroauric ions were initially bound and reduced to sub-10 $\mathrm{nm}$ Au nanoparticles on the positively charged CNF surfaces within the fast nucleation stage of $<10 \mathrm{~min}$. 
A
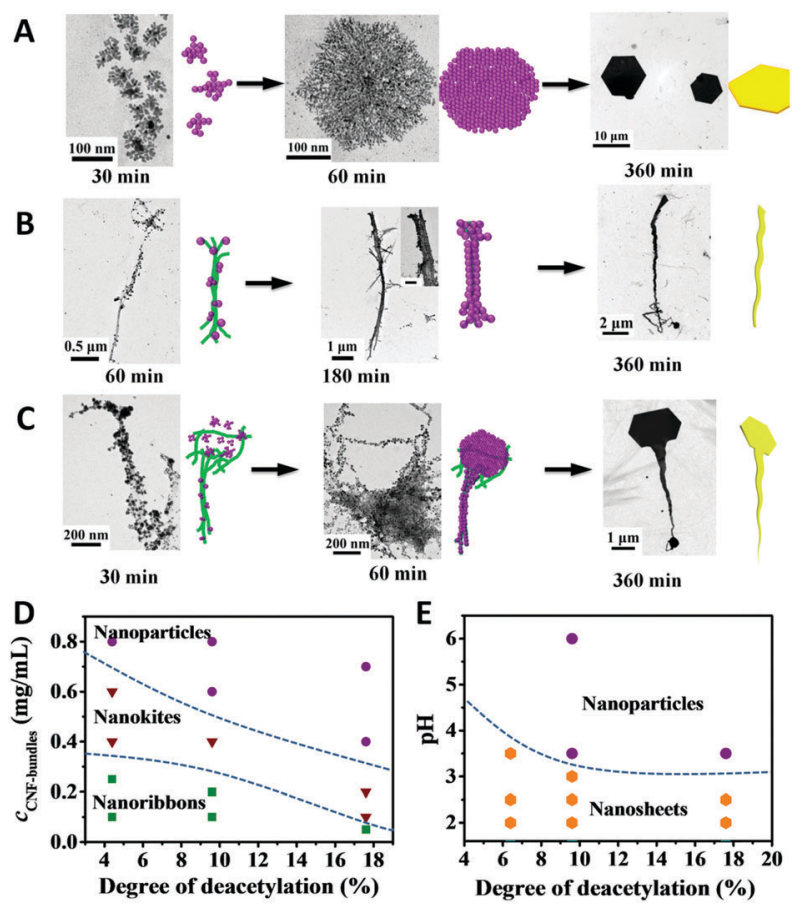

Fig. 2 Growth mechanisms and experimental parameters of Au nanocrystals. (A) Growth of Au nanosheets guided by CNFs. (B) Growth of Au nanoribbons guided by CNF-bundles. (C) Growth of Au nanokites guided by CNF-bundles. (D) Synthesis conditions of Au nanocrystals from CNFbundles at $\mathrm{pH}$ 3.5. (E) Synthesis conditions of Au nanocrystals from CNFs (0.08 wt\%).

During the slow growth process, these nanoparticles further grew and aggregated into dendritic quasi-hexagons or quasitriangles though Oswald ripening in order to lower their surface energies, ${ }^{9,21}$ which finally fused into well-defined Au nanosheets with the $\{111\}$ surfaces capped by a high density of CNFs (Fig. S8C, ESI $\dagger$ ).

In the suspension of CNF-bundles, chloroauric ions were also preferentially bound and reduced on the surfaces of the CNF-bundles during the nucleation stage. However, in comparison to those in the solution of CNFs, the obtained $\mathrm{Au}$ nanoparticles were much larger and heterogeneously dispersed (Fig. S9A, ESI $\dagger$ ) because the CNF-bundles may offer localized reduction reinforcement. During the subsequent growth process, the CNF-bundles further spatially guided the formation of Au nanoparticles and their aggregating states, depending on their concentration (Fig. 2D). At the lower concentration of CNF-bundles (Fig. 2B and Fig. S9B, ESI $\dagger$ ), certain individual CNF-bundles were nearly completely covered by $\mathrm{Au}$ nanoparticles, which thus tended to fuse into nanoribbons capped with nanofibrils to reduce their surface energies. Upon increasing the concentration of CNF-bundles (Fig. 2C and Fig. S9C, ESI $\dagger$ ), large aggregates of CNF-bundles may provide local microenvironments to concentrate $\mathrm{Au}$ nanoparticles along thicker rods or in large irregular domains. Thus there appears to be a probability of forming wider Au ribbons (see Fig. S9D, ESI $\dagger$ ), as well as Au nanokites with regular nanosheets as the heads and nanoribbons as the tails (Fig. S9E, ESI $\dagger$ ). However, a rapid reducing effect at too-high concentrations could drive the growth of Au nanomaterials away from a "kinetically controlled" pathway, thus leading to the formation of large Au nanoparticles (Fig. S10A, $\mathrm{ESI} \dagger$ ). Besides the exfoliation degree and concentrations of chitin nanofibrils, the morphologies of the Au nanocrystals could be further modulated by the deacetylation degree and $\mathrm{pH}$ values. For instance, 2D Au morphologies (nanoribbons \& nanokites) tended to form at moderate deacetylation degrees and lower $\mathrm{pH}$ (Fig. 2D, and E and Fig. S10B, and S11, ESI $\dagger$ ).

Considering that the $\{111\}$ crystallographic planes of $\mathrm{Au}$ nanocrystals are normally unfavoured by thermodynamics, ${ }^{22,23}$ the strong capping effect of the chitin nanofibrils was also essential to stabilize the $2 \mathrm{D}$ Au nanocrystals. Ultimately a large amount of chitin nanofibrils adhered densely to the resultant $\mathrm{Au}$ nanocrystals (Fig. S12, ESI $\dagger$ ), which even remained after 6 cycles of precipitating and washing. On account of the incompatibility hindrances of inorganic nanomaterials in developing inorganicorganic hybrids, ${ }^{24,25}$ the strong adhesion of chitin nanofibrils to $\mathrm{Au}$ surfaces may open up an unprecedented scenario to combine $\mathrm{Au}$ nanocrystals with organic materials. For example, a flexible green circuit could be prepared by sequentially filtering suspensions of gold nanosheets and chitin nanofibrils with the assistance of a patterned mask (Fig. 3A and Fig. S13A, ESI $\dagger$ ). The conductive layer ( $200 \mathrm{~nm}$ ) consisted of Au nanosheets capped with CNFs (Fig. 3B) and had a conductivity that was tuneable from insulating to metallic conductivity by washing these $\mathrm{Au}$ nanosheets for different times. The chitin substrate exhibited high mechanical flexibility and strength (e.g. $E=4.1 \mathrm{GPa}$ and $\sigma_{\mathrm{b}}=57.5 \mathrm{MPa}$ in Fig. S13B, ESI $\dagger$ ).

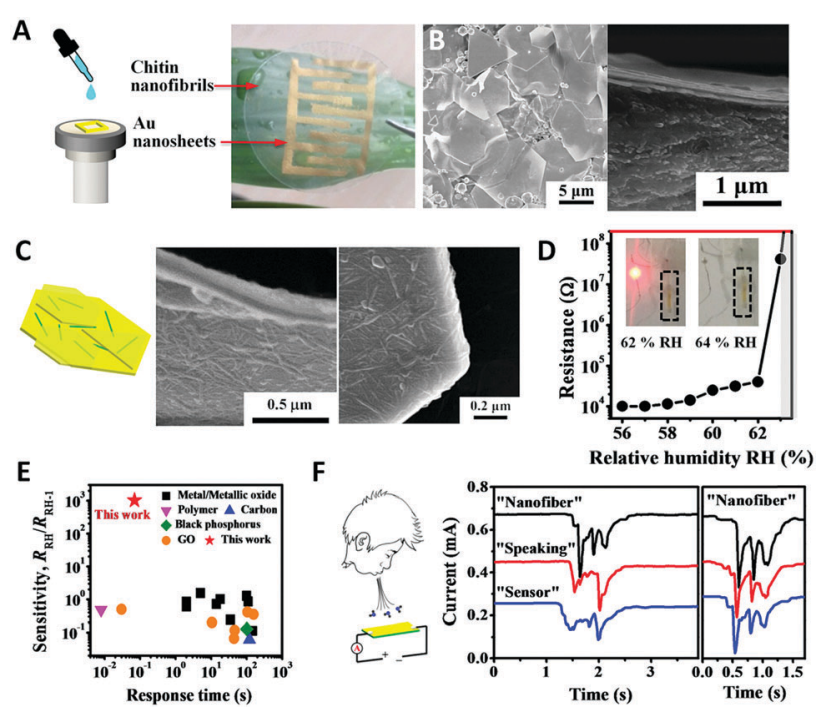

Fig. 3 Flexible hybrid circuits produced from Au nanosheets for high humidity-sensitivity. (A) Patterning Au nanosheets on a film of CNFs via sequential filtration. (B) Surface (left) and cross-section (right) SEM images of the hybrid circuit. (C) Layered intercalation of CNFs between Au nanosheets in the hybrid circuit. The CNFs adhered densely to the Au nanosheets. (D) Humidity-sensing evaluation via the electric conductivity of the hybrid circuit. The insets show the transition from conductivity to insulation induced by enhancing the humidity from $62 \%$ to $64 \%$. (E) The sensing properties of the resultant hybrid circuit in comparison with various humidity sensors in the literature. (F) Breath sensing evaluation via the electric conductivity during speaking different words. 
Because of strong mutual H-bonding of the chitin nanofibrils, the CNFs adhering to the Au nanosheets not only ensured strong interactions between the $\mathrm{Au}$ nanosheets and the chitin substrate, but also afforded the electric circuit with an ultrafast and ultrasensitive response to relative humidity $(\mathrm{RH})$. Since the conductive layer consists of intercalated layers of gold nanosheets and chitin nanofibrils, the presence of hydrophilic CNFs is expected to allow the diffusion of water and swelling, which can thus destroy the contacts between gold nanosheets (Fig. 3C and Fig. S13C, ESI $\dagger$ ). Higher humidity means a higher swelling ratio, and thus gradually decreases the conductive path among gold nanosheets. As a result, the resistance of the conductive layer increased with an increase of surrounding humidity. Specifically, the hybrid circuit showed an abrupt increase of electric resistance up to $>3$ orders of magnitude when enhancing the $\mathrm{RH}$ value beyond $62 \%$ (Fig. 3D and Fig. S14A, ESI $\dagger$ ). As a humidity responsive device, its resistance change ratio characterized by $R_{\mathrm{RH}+1} / R_{\mathrm{RH}}$ could reach an unprecedented value of 1019 (Fig. 3E and Table S1, ESI $\dagger$ ). ${ }^{6,26-29}$ In addition to super response repeatability and reversibility (Fig. S14B, ESI $\dagger$ ), its response time also reached as low as $10^{-1} \mathrm{~s}$. When placed close to the mouth (Fig. 3F and Fig. S14C, ESI $\dagger$ ), this sensor was able to precisely sense speech by measuring the variation of electric current and therefore breath humidity. Different patterns of electric current represent different breath strengths in spoken words, e.g. "nanofibers", "speaking", and "sensor".

Besides $\mathrm{Au}$ nanosheets for the construction of sensing hybrids, Au nanoribbons capped with chitin nanofibrils could also be employed to fabricate conductive cellulose hybrids with low electrical percolation thresholds. In an idealized composite built up from freely overlapping nanowires randomly placed in a continuous matrix (Fig. S15A-C, ESI $\dagger$ ), the electrical percolation threshold $\left(P_{\mathrm{c}}\right)$ correlates with the aspect ratio $(L / D)$ of fillers as $P_{\mathrm{c}} \sim(L / D)^{-2} 27 \pi / 4$ according to the Interparticle Distance model, ${ }^{30,31}$ which gives a $P_{\mathrm{c}}$ value within $5.3-0.43$ vol\% in the case of $L / D$ within 20-70. On account of their broad distribution of $L / D$ as well as the ribbon morphology and geometric flexibility, the synthesized Au nanoribbons gave a high $P_{\mathrm{c}}$ value of 6.9 vol\% in the chitin matrix (Fig. S15E, ESI $\dagger$ ).

On the other hand, it was suggested that conductivity could be retained in a porous composite by organizing low-aspect-ratio conductive fillers in elongated directions. ${ }^{32}$ Thus we endeavoured to minimize the amount of $\mathrm{Au}$ nanoribbons in conductive composites by filtering $\mathrm{Au}$ nanoribbons through porous tissue paper (Fig. 4A and B). Due to strong H-bonding of the chitin nanofibrils with polyhydroxyl surfaces, ${ }^{33}$ the Au nanoribbons adhered and formed conductive networks on the surfaces of cellulose fibres (Fig. 4C and Fig. S16A, ESI $\dagger$ ). Only 0.09 vol\% Au nanoribbons (taking void into account) could achieve a surface resistance of $\sim 220 \Omega \mathrm{sq}^{-1}$ in tissue paper, which to our knowledge has not been observed before in other reported composites (Fig. 4D). ${ }^{6-8,34,35}$ This conductive tissue paper was able to maintain its conductivity during the processes of twisting, bending, washing and heating (Fig. S17, ESI $\dagger$ ). Moreover, the conductive performance of the tissue paper could be easily tuned from being a uniform conductor to a Janus-faced conductor, according to the
A
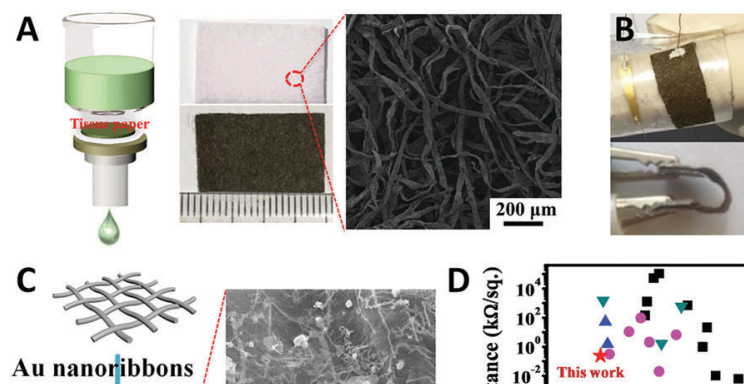
coating
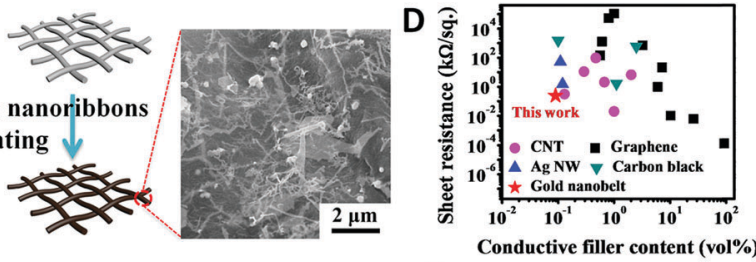

E
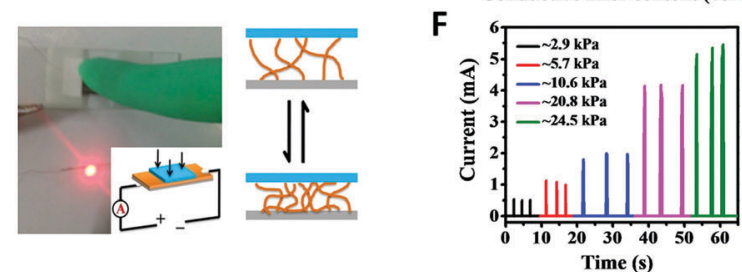

Fig. 4 Conductive fabric produced from Au nanoribbons for high pressure-sensitivity. (A) Filtering in Au nanoribbons into tissue paper (top middle image) for conductive fabric (bottom middle image) via filtration. (B) Flexibility evaluation. (C) Conductive networks of Au nanoribbons on the surfaces of cellulose fibres. (D) Sheet resistance versus filler content of the conductive fabric in comparison with conductive composites with different fillers (indicated by different symbols). (E) A schematic illustration of the pressure-sensing setup. (F) The pressure-induced current response of the conductive fabric.

adsorbed content of Au nanoribbons (Fig. S16B, ESI $\dagger$ ). In the case of the Janus-faced conductive tissue paper, the top-side was highly conductive due to the coating of the abundant and continuous gold network, while the bottom-side was insulated because its surface only adsorbed a small amount of discrete gold nanoribbons (Fig. S16C, ESI $\dagger$ ). When applying pressure (on $0.8 \times 1.2 \mathrm{~cm}^{2}$ ), its conductance showed a reproducible pressure-dependent behaviour (Fig. 4E and F), because of an increase in the number of physical contacts among the cellulose fibres. As a pressure-sensing device, it had a response time as low as $6.2 \mathrm{~ms}$ and nearly $100 \%$ recovery in $\sim 8.1 \mathrm{~ms}$ (Fig. S18C-E, ESI $\dagger$ ). The gauge factor (GF) was $0.97 \mathrm{kPa}^{-1}$ for a pressure range within 2.9-10.1 $\mathrm{kPa}$ and $0.13 \mathrm{kPa}^{-1}$ for 10.1-24.5 kPa, being comparable with other pressure sensors (Fig. S18b and Table S3, ESI $\dagger$ ).

More interestingly, as illustrated in Fig. S19A (ESI $\dagger$ ), by integrating the gold-derived humidity sensor and pressure sensor on the surface of a flexible PDMS substrate, a logic circuit could be easily prepared, e.g., a 2, 3 linkage for the 'AND' logic gate and a 2, 3 linkage for the 'OR' logic gate. These circuits responded quite quickly to environmental conditions, providing output characteristics of 'OR' and 'AND' circuits. For example, a circuit based on the 'AND' gate generated a high signal at output (the ' 1 ' state) when the aridity was high and the external pressure was applied (the ' 1,1 ' state in Fig. S19B, ESI $\dagger$ ), and the signal at the output was low (' 0 ') when at least one of the two signals was not given. On the contrary, by using the 'OR' configuration, the same sensing unit demonstrated a ' 0 ' 
for the '0,0' input and ' 1 ' for all other input values (Fig. S19C, ESI $\dagger$ ). In addition, the logic circuit was flexible and could be adhered to skin, suggesting utility for electronic skins and biosensors (Fig. S19D and E, ESI $\dagger$ ).

\section{Conclusions}

In summary, as low-cost and widely-available marine nanomaterials, chitin nanofibrils were found to be capable not only of binding and reducing chloroauric ions on their deacetylated surfaces, but also of guiding the growth direction of $2 \mathrm{D} \mathrm{Au}$ nanocrystals into various geometries (i.e. sheets, kites and ribbons) via different exfoliated states. During this "kinetically controlled" mineralization process, chitin nanofibrils served simultaneously as the reductant, capping agent and soft template of the Au nanocrystals, analogously to protein-involved mineralization. Due to the capping effect of chitin nanofibrils on the $\{111\} \mathrm{Au}$ facets, the chitin nanofibrils could also adsorb tightly to $\mathrm{Au}$ nanoribbons and nanosheets, which afforded them with strong adhesion to polyhydroxy surfaces and thus made ideal building blocks for various wearable sensing hybrids potentially for smart clothing, human-machine interfacing devices and multifunctional electronic skins.

\section{Conflicts of interest}

There are no conflicts to declare.

\section{Acknowledgements}

The National Natural Science Foundation of China (No. 21474125, No. 51603224), the Chinese "1000 Youth Talent Program", the Shandong "Taishan Youth Scholar Program", the Natural Science Foundation of Shandong Province (JQ201609), and the Shandong Collaborative Innovation Centre for Marine Biomass Fiber Materials and Textiles are kindly acknowledged for their financial support.

\section{References}

1 S. Mann, Nature, 1988, 332, 119-124.

2 F. Nudelman, K. Pieterse, A. George, P. H. H. Bomans, H. Friedrich, L. J. Brylka, P. A. J. Hilbers, G. de With and N. A. J. M. Sommerdijk, Nat. Mater., 2010, 9, 1004-1009.

3 G. Falini and S. Fermani, Tissue Eng., 2004, 10, 1-6.

4 Z. Fan and H. Zhang, Acc. Chem. Res., 2016, 49, 2841-2850.

5 X. Hong, C. Tan, J. Chen, Z. Xu and H. Zhang, Nano Res., 2015, 8, 40-55.

6 C. Li, S. Bolisetty and R. Mezzenga, Adv. Mater., 2013, 25, 3694-3700.

7 J. Zhou, A. Saha, J. Adamcik, H. Hu, Q. Kong, C. Li and R. Mezzenga, Adv. Mater., 2015, 27, 1945-1950.

8 S. Gong, W. Schwalb, Y. Wang, Y. Chen, Y. Tang, J. Si, B. Shirinzadeh and W. Cheng, Nat. Commun., 2014, 5, 3132 .

9 C. Zhu, H.-C. Peng, J. Zeng, J. Liu, Z. Gu and Y. Xia, J. Am. Chem. Soc., 2012, 134, 20234-20237.
10 C. M. Payne, D. E. Tsentalovich, D. N. Benoit, L. J. E. Anderson, W. Guo, V. L. Colvin, M. Pasquali and J. H. Hafner, Chem. Mater., 2014, 26, 1999-2004.

11 H. L. Qin, D. Wang, Z. L. Huang, D. M. Wu, Z. C. Zeng, B. Ren, K. Xu and J. Jin, J. Am. Chem. Soc., 2013, 135, 12544-12547.

12 L. Y. Wang, X. Chen, Y. C. Chai and J. C. Hao, Colloids Surf., A, 2007, 293, 95-100.

13 N. Zhao, Y. Wei, N. Sun, Q. Chen, J. Bai, L. Zhou, Y. Qin, M. Li and L. Qi, Langmuir, 2008, 24, 991-998.

14 B. Lim, P. H. C. Camargo and Y. Xia, Langmuir, 2008, 24, 10437-10442.

15 L. Au, B. Lim, P. Colletti, Y. S. Jun and Y. Xia, Chem. - Asian J., 2010, 5, 123-129.

16 J. Huang, L. Lin, D. Sun, H. Chen, D. Yang and Q. Li, Chem. Soc. Rev., 2015, 44, 6330-6374.

17 S. Bolisetty and R. Mezzenga, Nat. Nanotechnol., 2016, 11, 365-371.

18 Y. Fan, T. Saito and A. Isogai, Carbohydr. Polym., 2010, 79, 1046-1051.

19 J. Zhang, J. Du, B. Han, Z. Liu, T. Jiang and Z. Zhang, Angew. Chem., Int. Ed., 2006, 45, 1116-1119.

20 H. Hu, J. Zhou, Q. Kong and C. Li, Part. Part. Syst. Charact., 2015, 32, 796-808.

21 G. Lin, W. Lu, W. Cui and L. Jiang, Cryst. Growth Des., 2010, 10, 1118-1123.

22 D. Wei, W. Qian, Y. Shi, S. Ding and Y. Xia, Carbohydr. Res., 2007, 342, 2494-2499.

23 L. Chen, I. Polishchuk, E. Weber, A. N. Fitch and B. Pokroy, Cryst. Growth Des., 2016, 16, 2972-2978.

24 S. K. Kumar, B. C. Benicewicz, R. A. Vaia and K. I. Winey, Macromolecules, 2017, 50, 714-731.

25 S. Neyshtadt, J. P. Jahnke, R. J. Messinger, A. Rawal, T. Segal Peretz, D. Huppert, B. F. Chmelka and G. L. Frey, J. Am. Chem. Soc., 2011, 133, 10119-10133.

26 J. Feng, L. Peng, C. Wu, X. Sun, S. Hu, C. Lin, J. Dai, J. Yang and Y. Xie, Adv. Mater., 2012, 24, 1969-1974.

27 M. A. Squillaci, L. Ferlauto, Y. Zagranyarski, S. Milita, K. Mullen and P. Samori, Adv. Mater., 2015, 27, 3170-3174.

28 D. H. Ho, Q. Sun, S. Y. Kim, J. T. Han, H. Kim do and J. H. Cho, Adv. Mater., 2016, 28, 2601-2608.

29 Q. Kuang, C. Lao, Z. L. Wang, Z. Xie and L. Zheng, J. Am. Chem. Soc., 2007, 129, 6070-6071.

30 E. J. Garboczi, K. A. Snyder, J. F. Douglas and M. F. Thorpe, Phys. Rev. E: Stat. Phys., Plasmas, Fluids, Relat. Interdiscip. Top., 1995, 52, 819-828.

31 J. Li, P. C. Ma, W. S. Chow, C. K. To, B. Z. Tang and J. K. Kim, Adv. Funct. Mater., 2007, 17, 3207-3215.

32 Y. Kim, J. Zhu, B. Yeom, M. Di Prima, X. Su, J.-G. Kim, S. J. Yoo, C. Uher and N. A. Kotov, Nature, 2013, 500, 59-63.

33 Y. Huang, M. Yao, X. Zheng, X. Liang, X. Su, Y. Zhang, A. Lu and L. Zhang, Biomacromolecules, 2015, 16, 3499-3507.

34 H. Nassira, A. Sanchez-Ferrer, J. Adamcik, S. Handschin, H. Mahdavi, N. Taheri Qazvini and R. Mezzenga, Adv. Mater., 2016, 28, 6914-6920.

35 G. Y. Han, B. Guo, L. W. Zhang and B. S. Yang, Adv. Mater., 2006, 18, 1709-1712. 